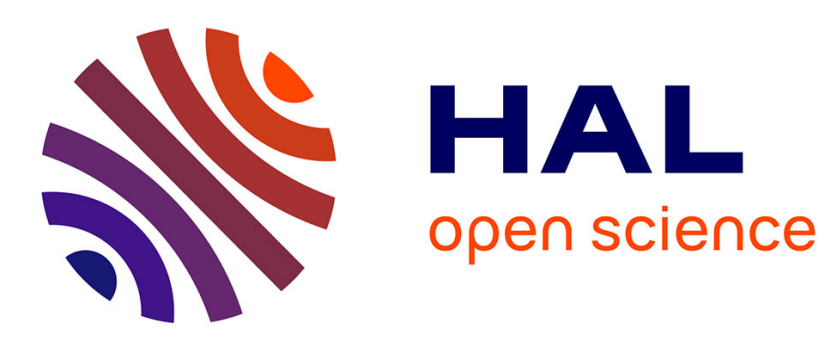

\title{
Compensated Microsphere-Assisted Interference Microscopy
}

\author{
Stéphane Perrin, Yidenekachew J Donie, Paul Montgomery, Guillaume \\ Gomard, Sylvain Lecler
}

\section{- To cite this version:}

Stéphane Perrin, Yidenekachew J Donie, Paul Montgomery, Guillaume Gomard, Sylvain Lecler. Compensated Microsphere-Assisted Interference Microscopy. Physical Review Applied, 2020, 13 (1), 10.1103/PhysRevApplied.13.014068 . hal-03084634

\section{HAL Id: hal-03084634 https://hal.science/hal-03084634}

Submitted on 21 Dec 2020

HAL is a multi-disciplinary open access archive for the deposit and dissemination of scientific research documents, whether they are published or not. The documents may come from teaching and research institutions in France or abroad, or from public or private research centers.
L'archive ouverte pluridisciplinaire HAL, est destinée au dépôt et à la diffusion de documents scientifiques de niveau recherche, publiés ou non, émanant des établissements d'enseignement et de recherche français ou étrangers, des laboratoires publics ou privés. 


\title{
Compensated Microsphere-Assisted Interference Microscopy
}

\author{
Stephane Perrin $\odot,{ }^{1, *}$ Yidenekachew J. Donie $\odot,{ }^{2,3}$ Paul Montgomery, ${ }^{1}$ Guillaume Gomard,, 3 \\ and Sylvain Lecler ${ }^{1}$ \\ ${ }^{1}$ ICube Laboratory, CNRS - INSA de Strasbourg —University of Strasbourg, 300 Boulevard Sébastien Brant, \\ 67412 Illkirch, France \\ ${ }^{2}$ Light Technology Institute, Karlsruhe Institute of Technology, Engesserstrasse 13, 76131 Karlsruhe, Germany \\ ${ }^{3}$ Institute of Microstructure Technology, Karlsruhe Institute of Technology, Hermann-von-Helmholtz-Platz 1, \\ 76344 Eggenstein-Leopoldshafen, Germany
}

\begin{abstract}
We propose an experimental method in microsphere-assisted interference microscopy that makes it possible to reconstruct surface topographies as much with high quantitative depth accuracy as when the transversal feature sizes are smaller than the classical diffraction limit. The full-field super-resolution interference microscope consists not only of one glass microsphere in the object arm but also of a second similar microsphere in the reference arm. By compensating the sphere aberrations in the two interference arms, the spatial resolution appears to be considerably increased. The increase in the three-dimensional spatial resolution allows for the topography reconstruction of 300-nm-width grating lines and 200-nm-diameter transparent nanopillars.
\end{abstract}

\section{INTRODUCTION}

Interference microscopy is a widely used noncontact optical technique enabling the reconstruction of the surface topography of microscopic objects [1] for surface-quality inspection or surface-roughness measurement. This fullfield imaging technique, in which the lateral measurement field (ranging from a few hundred micrometers to several millimeters) depends on the combination of the microscope objective performance and the sensor chip size, is able to achieve a high axial sensitivity (approximately $5 \mathrm{~nm}$ ). However, interference microscopy has a lateral resolving power that is not only limited by the diffraction of light [as in the case of two-dimensional (2D) optical microscopy] [2] but also by its ability to reconstruct the height map at the same time [3]. Indeed, characterizing the performance of an instrument in order to retrieve the surface topography is more complex than simply estimating its 2D imaging performance [4]. According to ISO 25178-600, the lateral-period limit of the topographic system is defined as the spatial period of a sinusoidal profile at which the height response of an instrument falls to $50 \%$ [5]. Therefore, the depth accuracy depends on the lateral feature size. Typically, when the spatial frequency of the object appears to be close to $\lambda$ (the wavelength of

\footnotetext{
*stephane.perrin@unistra.fr
}

light), the instrument response related to the depth measurement can no longer be considered as linear $[4,6]$. Combined with optical aberrations as well as the influence of the nature of light $[7,8]$, the lateral resolution in three-dimensional (3D) surface profilometry is usually not higher than a few micrometers, hence considerably increasing the nonisotropic 3D spatial resolution. Recently, several techniques have been proposed to improve the lateral-resolution ability of 3D microscopy, for example, the development of structured-illumination 3D profilometry [9] and of tomographic diffractive microscopy [10,11]. In 2009 and in 2011, 2D super-resolution imaging was experimentally demonstrated in air using nanoscale spherical lenses [12] and glass microspheres [13], respectively.

Microsphere-assisted microscopy is a super-resolution imaging technique that has the advantage of being easy to implement, label free, full field, and fast [14]. The technique consists in introducing a transparent microsphere, having a diameter ranging from $5 \mu \mathrm{m}$ to $150 \mu \mathrm{m}$, in the imaging part of a classical white-light microscope. The microsphere acts as a magnifying optical component [15], generating a super-resolved image that is collected by an objective lens of the microscope. Geometrical (e.g., the microsphere diameter) and optical (e.g., the illumination wavelength and refractive index of the microsphere) parameters thus have an influence on performance. For example, the nature of the image can be virtual or real depending on the contrast refractive index $[16,17]$. A lateral resolution of $100 \mathrm{~nm}$ has been claimed 
in air $[13,18]$, thus being equivalent to the resolution limit in structured-illumination microscopy and in tomographic diffractive microscopy. Nevertheless, the phenomenon behind the super-resolution remains not fully understood. It has been reported that the microsphere collects the nearfield information of the sample and then propagates it into the far field $[19,20]$. The immersion of high-refractiveindex microspheres in a surrounding medium has also been suggested to improve the imaging quality [21,22]. More recently, microsphere-aided microscopy has been combined with dark-field illumination for the label-free visualization of translucent samples [23] and with interferometry for the high-resolution reconstruction of surface topography $[24,25]$ or of cell volume [26]. These previously reported methods in microsphere-aided interference microscopy have been implemented by placing a glass microsphere in the object arm of a quantitative-phase interferometer, leading, first, to an unbalanced optical path difference between the arms and, second, to the generation of optical aberrations, as highlighted in Ref. [25]. An imageprocessing method based on $2 \mathrm{D}$ data fitting has thus been required to remove the spherical component. Since then, it has been implemented assuming that the wave-front deformation provided by the microsphere is well known and that the microsphere is of high quality. However, at this scale, it appears to be complex to retrieve wave-front deformation by a microsphere. The image-processing algorithm, more complex to automate, is thus individually implemented, leading to a long processing time (e.g., about $20 \mathrm{~min}$ in our case, depending on the performance of the computer and the image sampling). Furthermore, despite an increase in the spatial resolution, the reconstruction of surface topography with a high height accuracy (e.g., reconstruction of the straight shapes of grating grooves) still remains a real challenge.

The method that we propose in this work allows for an accurate 3D profile reconstruction for straight shapes of grating grooves. Our microsphere-assisted interference microscope consists not only of one microsphere in the object arm, but also of a second similar microsphere in the reference arm. In this way, the wave-front distortion due to the optical aberrations is avoided and the optical path difference in the interferometer arms is compensated. The performance of compensated-dispersion microsphereaided interference microscopy is demonstrated in air using a coherent phase-shifting interference microscope in a Linnik configuration and in a reflection mode. Such a method has the advantage of not requiring the implementation of image processing, thus being of further promise in super-resolution surface metrology [27]. The areal topography of features having a spatial period smaller than $\lambda$ is reconstructed with high axial and lateral accuracy $[4,5]$. We demonstrate that the imaging system can retrieve the details with a high-spatial resolution through nanometric axial sensitivity.

\section{METHOD}

Figure 1 shows the layout of the microsphere-assisted interference microscope in a Linnik configuration, thus allowing for the collection of the back-reflected wave fronts from both the sample and the reference mirror. The emitting beam from the light source (LED, $\lambda_{0}=520 \mathrm{~nm}$, $\Delta \lambda=31 \mathrm{~nm}$ ) passes through a series of collimating and focusing lenses, as well as a pinhole for spatial filtering. The illumination beam is thus spatially coherent and temporally quasicoherent in order to reduce speckle generation and coherent noise [28]. With regard to the incident beam, $50 \%$ is reflected into the reference arm and $50 \%$ is transmitted into the object arm by a beam-splitter cube. Given the requirement of two similar microspheres for dispersion compensation, the object arm is therefore tilted at $90^{\circ}$ by a mirror in order to have a horizontal object plane for easier placement of the compensating microsphere. In this optical setup, the two microspheres are first randomly deposited on the surfaces of the object and the reference mirror. Despite being in contact with the sample, the latter is, however, not affected due to the light microsphere. In order to perform noninvasive measurements, an optical head can be introduced, allowing the microsphere to

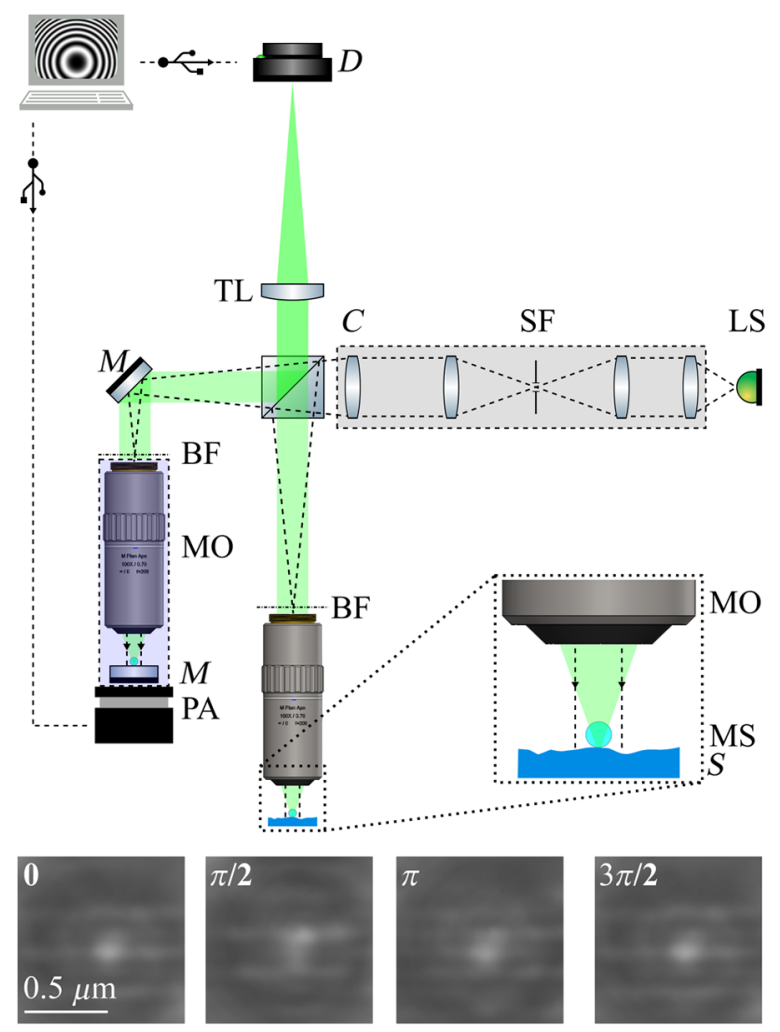

FIG. 1. The layout of the super-resolution interference microscope: LS, light source; SF, spatial filtering; C, condenser lens; $\mathrm{MO}$, microscope objective; BF, back focal plane; S, sample; M, mirror; MS, microsphere; PA, piezoelectric actuator; TL, tube lens; D, detector. 
be held at a distance (less than the wavelength) from the surface [29-31]. $X-Y$ stages are used in the reference arm to match the transverse positions of the two similar microspheres. In each arm of the interferometer, the coupled microscope objective and microsphere allow the super-resolution phenomenon to be achieved with the same resolving power. The two microscope objectives (Mitutoyo Corporation) have a magnification factor of $\times 50$ and a numerical aperture of 0.55 . The condenser lens, placed in an afocal configuration with the microscope objectives, enables wide-field illumination of the microspheres. The soda-lime-glass microspheres (Cospheric LLC) have a diameter of $30 \mu \mathrm{m}$ and a refractive index of 1.52 . The diameter dispersion of the microspheres is guaranteed to be less than $10 \%$ and the sphericity to be more than $95 \%$. The dispersion range is decreased by selecting only the same microspheres through the microscope objectives of the object and reference arms. This method leads to an error of the diameter dispersion of $\pm 300 \mathrm{~nm}$. Moreover, before performing a measurement, the surface of the microspheres is examined through the microscope objectives. In doing so, microspheres having imperfections or scratches (with a size larger than the resolution of the objectives) on the bottom are excluded. However, it appears to be complex to estimate the surface quality of the rear side of the microsphere. The selection of good microspheres is less time consuming than the implementation of image processing when only one microsphere is used in the object arm. Also, the selection needs to be applied only one time. The objective lenses then collect the virtual images of both the sample and the mirror, generated by the microspheres in the object and reference arms, respectively. The object and reference beams are superimposed by the beam-splitter cube. At the output of the interferometer, a tube lens, which forms an infinity-corrected optical system, finally directs the super-resolved images onto the detector (Basler AG). The lateral magnification factor from the microsphere reaches 4.5 , as previously estimated [15]. In the object plane, the size of the square pixels is equal to $31.5 \mathrm{~nm}$, giving a high spatial sampling of the object features. To implement the $\pi / 2$ phase shift, all the optical elements of the reference arm are axially displaced using a piezoelectric actuation system. In our case, the $\pi / 2$ phase shift should lead to an increment of the piezoelectric device of $66.25 \mathrm{~nm}$ [1]. However, in the presented results, the high numerical apertures of the microspheres must be considered in the axial increment to prevent wrong interpretations of the depth results [32]. This not being trivial with regard to microspheres (it is complex to estimate their numerical aperture), a specific calibration of the phase shifter has previously been made using a $1200-\mathrm{nm}$-period standard. Through a $30-\mu \mathrm{m}$-diameter microsphere in air, the $\pi / 2$ phase shift now corresponds to an axial increment of $78 \mathrm{~nm}$. The coherence function (or visibility) of the light source is constant at least within the axial stepping range. The series of interferograms make it possible to retrieve the phase-difference distribution between the object and the flat reference using a four-step phaseshifting algorithm [1]. Fig. 1 shows the four interferograms used for the surface-topography reconstruction presented in Fig. 4(b). In addition, a 2D unwrapping algorithm based on least squares is implemented to remove the $2 \pi$ jumps [33], which result from the arctan function.

\section{RESULTS}

Figure 2 shows the transfer function of a $\times 50$ magnification microscope objective alone (without using microspheres) of the Linnik-based interference microscope as a function of the object transverse frequency. A positive Ronchi ruling with a period ranging from $5 \mu \mathrm{m}$ to $200 \mathrm{~nm}$ is placed in the object arm of the interferometer. The shape of the contrast evolution corresponds to the shape of an aberration-free quasicoherent imaging system [7]. The objective lenses are thus assumed to be diffraction limited and the lateral-resolution limit equals $950 \mathrm{~nm}$ in air in the absence of microspheres. However, in interference microscopy, the lateral resolution depends not only on the transfer function of the transverse frequencies but also on its ability to transfer height information $[3,4,6]$. In our case, the Linnik-based interference microscope alone is thus not able to reconstruct object features that have a period smaller than $1.8 \mu \mathrm{m}$, as shown in Fig. 3 . The line features of the $50 \%$-duty-cycle grating standard (SiMETRICS GmbH), which has a period of $2 \mu \mathrm{m}$ and a depth of $200 \mathrm{~nm}$, are indeed resolved, unlike the 600-nmperiod features. The height profiles in Fig. 3(c) highlight the resolution limit of the interference microscope without microspheres. The standard deviation $\sigma$ of the phase distribution of a flat plane reaches $21 \mathrm{mrad}$, i.e., the axial sensitivity of the interferometer is $1.8 \mathrm{~nm}$.

The addition of a $30-\mu \mathrm{m}$-diameter soda-lime microsphere in each interference arm considerably improves the lateral resolution of the imaging system. As such,

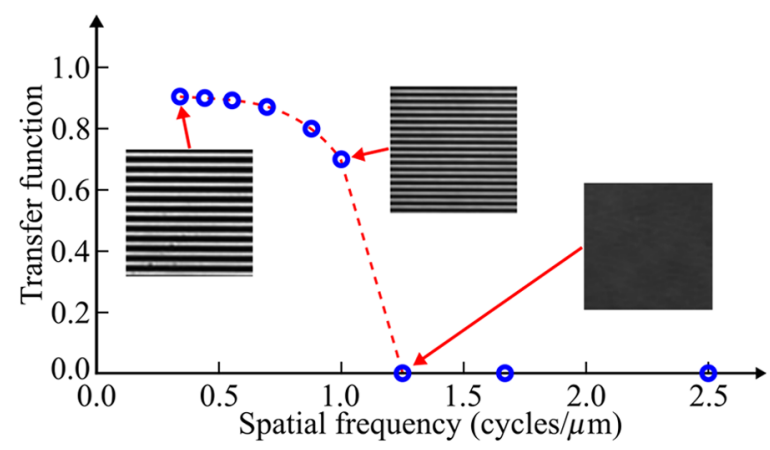

FIG. 2. The contrast transfer function of the object arm of the interference microscope without any microspheres. The lateral resolution is limited by diffraction of light and the cut-off frequency equals 1.25 cycles $/ \mu \mathrm{m}$. 
(a)
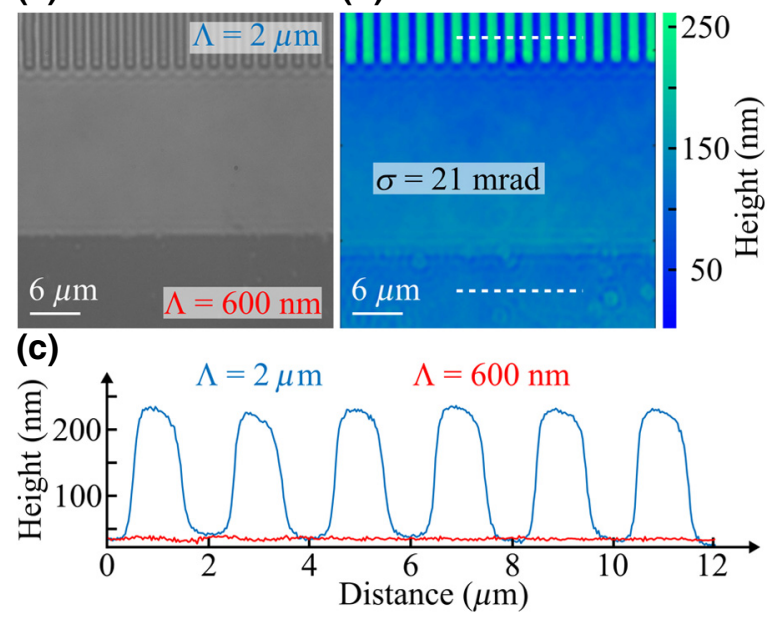

FIG. 3. The performance of the interference microscope without microspheres. (a) Direct images of $2-\mu \mathrm{m}$-period (at the top) and 600-nm-period (at the bottom) standards. (b) Surfacetopography reconstruction using the four-step phase-shifting algorithm. (c) The height profiles of the 2- $\mu \mathrm{m}$-period and 600$\mathrm{nm}$-period standards along the dashed white lines in (b).

the microsphere-assisted phase-shifting microscope is then able to reconstruct the 600 -nm-period grating of the standard in air. Since no postprocessing of the data is required to remove the phase deformation from the microsphere, phase measurement can be used directly. Figure 4(a) demonstrates the ability of the imaging system to retrieve the square shape of the grating lines (solid blue curve) with high accuracy over the majority of the measured area [Fig. 4(b)]. The measured heights, i.e., $140 \mathrm{~nm}$, are in agreement with the heights measured using atomic force microscopy (AFM), as shown by the dashed red curve in Fig. 4(a). However, while the results [Figs. 4(c.i) and 4(c.ii)] give the correct depth, AFM appears to be limited for the reconstruction of the straight shapes of the 300nm-width grooves. In fact, the convolution of the tip shape with the object results in a "V"'-shape reconstruction of the square features and a wrong duty cycle of $18 \%$. In fact, the width of the grooves of the standard equals the space between the grooves, as shown in Fig. 4(d) using scanning electron microscopy (SEM). In addition, the dotted black curve in Fig. 4(a) represents the depth profile measured with the microsphere-aided interference microscope having only one microsphere in the object arm, i.e., without aberration compensations. In this case, a $2 \mathrm{D}$ polynomial fit of the wave front is required to retrieve the topography. The axial sensitivity of the aberration-compensation interference nanoscope is slightly decreased when using two microspheres, going from $\sigma=1.8 \mathrm{~nm}$ (without any microspheres) to $\sigma=5.6 \mathrm{~nm}$. By applying a median filter to the interferograms, it is possible to reach a sensitivity of $3.9 \mathrm{~nm}$. The M-C curve, in Fig. 4(a), is plotted (a)
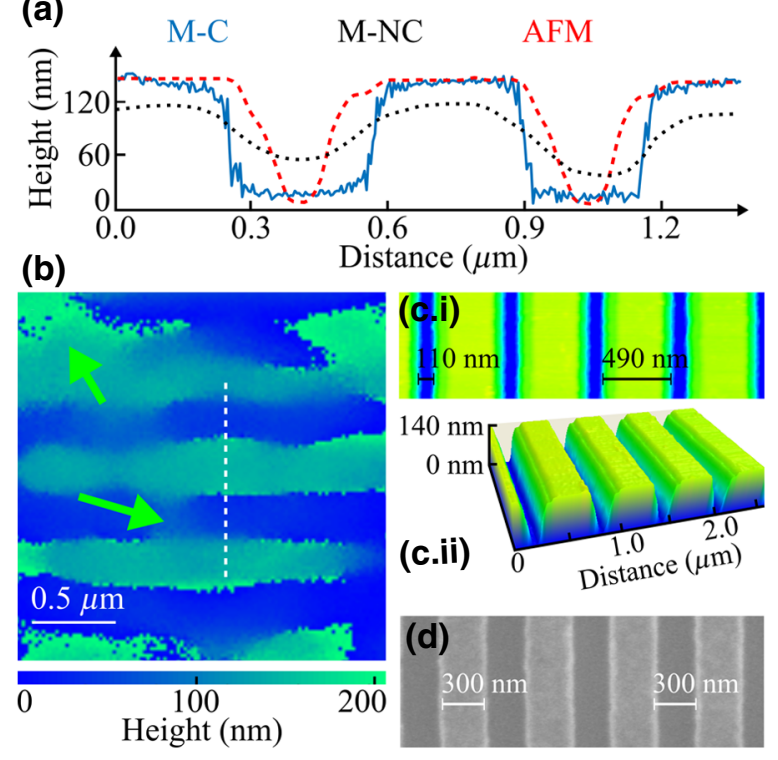

FIG. 4. The performance of the microsphere-aided interference microscope. (a) A comparison of the height profiles of the 600$\mathrm{nm}$-period standard using aberration-compensated microsphereaided interference microscopy (M-C, solid blue curve) along the dashed white lines in (b), noncompensated microsphereaided interference microscopy (M-NC, dotted black curve), and AFM (dashed red curve). (b) High-resolution surface-topography reconstruction. (c.i) 2D and (c.ii) 3D views of the AFM topography reconstruction of the 600-nm-period standard. (d) A SEM direct image.

using raw data, leading to a noisier signal than the two other methods, M-NC and AFM, where the data are filtered using a $3 \times 3$ median filter and averaged by the round-trip scanning mode, respectively. The higher-frequency noise of the M-C curve can mainly be explained by nonzero optical path differences between the two microspheres at each transverse position, i.e., lateral nanometric disparities. Also, the obliquely incident beams on a straight grating can be subject to multiple reflections before being backreflected and collected by the microsphere, increasing the optical path. By compensating the optical path difference in both arms, the super-resolution phenomenon is therefore enhanced in comparison with the previous results reported in Refs. [24-26]. Nevertheless, such a high-quality measurement contributes to the clear visibility of the areas of artifact generation [green arrows in Fig. 4(b)]. In fact, the defocused patches are most likely due to imperfections on the microsphere surface and inside the microsphere (despite the microspheres being previously selected to have the same diameter, a high sphericity, and a high surface quality), leading to distortions, a potential reduction of the lateral field of view, empty areas, or wrong height values. These microsphere imperfections have a hundredsof-nanometers order of magnitude, unlike the nanometric imperfections behind the high-frequency noise. 
The grating standards are then substituted for selfassembled and disordered nanopillars made of poly(methyl methacrylate), processed on a silicon substrate (Karlsruhe Institute of Technology, Germany). They are obtained by the polymer-blend-lithography method and developed as light-trapping substrates for photovoltaic applications (for more details, see Ref. [34]). The diameters of the obtuseangled nanopillars range from $200 \mathrm{~nm}$ to $450 \mathrm{~nm}$ and their average height is $80 \mathrm{~nm}$ (a non-negligible height dispersion, ranging from $70 \mathrm{~nm}$ to $100 \mathrm{~nm}$, remains). Figure 5(a) presents the direct image of the sample using AFM. The AFM technique needs an acquisition time of around $20 \mathrm{~min}$ to measure the surface topography of the sample even though, using microsphere-assisted phase-shifting microscopy, the height distribution of the nanopillars is quasi-immediately reconstructed [Fig. 5(b)]. The AFM and $\mathrm{M}-\mathrm{C}$ measurements are performed at different locations, due to the random arrangement of the sample. The nanopillars being transparent, light is delayed upon passing through them. This axial phase delay makes it possible to estimate the optical distance, which depends on both the height and the refractive index of the poly(methyl methacrylate)-based nanopillars $(n=1.48)$, and thus to retrieve the $3 \mathrm{D}$ height distribution of the nanopillars. A height dispersion of $86 \mathrm{~nm} \pm 12 \mathrm{~nm}$ is retrieved using the $\mathrm{M}-\mathrm{C}$ interferometer. The super-resolving power of the microsphere-aided interference microscope allows the phase delay of the small features to be measured with high (a)

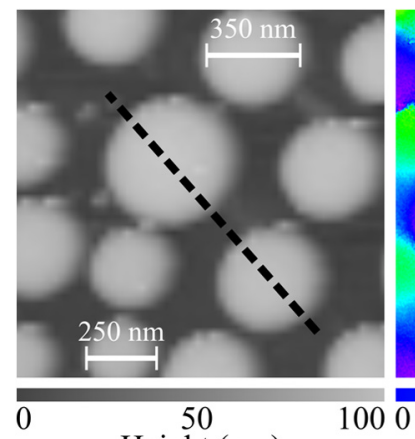

(c)

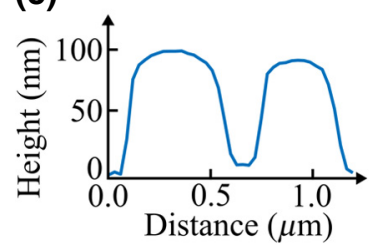

(b)

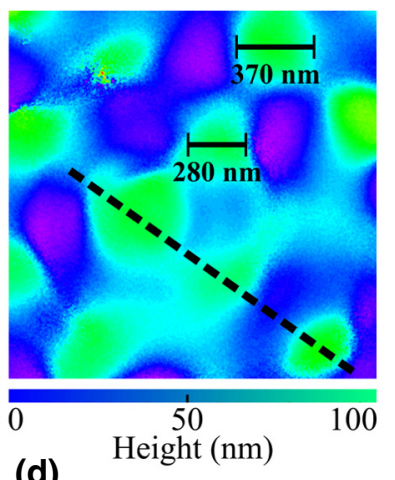

(d)

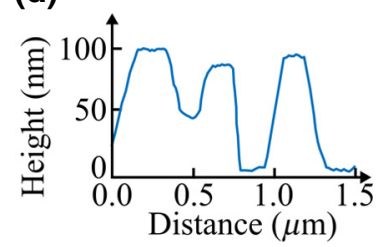

FIG. 5. The characterization of a disordered array of nanopillars using microsphere-assisted interference microscopy. (a) The topography using AFM. (b) High-resolution surface-topography reconstruction using M-C. The two topographies shown in (a) and (b) are not performed at the same position. (c),(d) Height profiles along the black dashed lines in (a) and (b), respectively. (a)
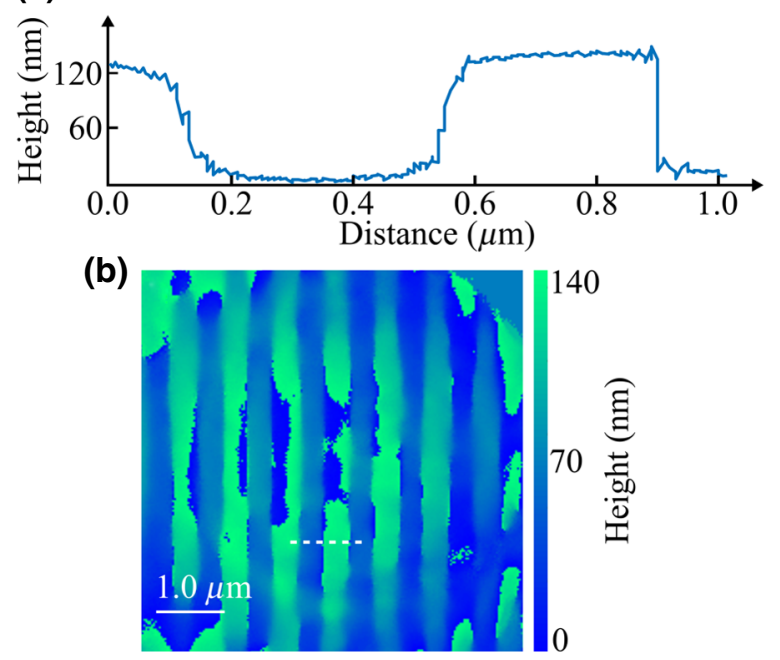

FIG. 6. The characterization of a 800-nm-period standard grating using microsphere-assisted interference microscopy compensated by two $50-\mu$ m-diameter microspheres. (a) The height profile along the dashed white lines of (b) the high-resolution surface-topography reconstruction.

accuracy, as shown via the height profiles in Figs. 5(c) and $5(\mathrm{~d})$.

\section{DISCUSSION}

Figure 6 shows the ability of compensated microsphereaided interference microscopy to retrieve the height distribution of a 50\%-duty-cycle standard grating (SiMETRICS $\mathrm{GmbH}$ ) using two microspheres having a diameter of $50 \mu \mathrm{m}$. Increasing the size of the microspheres allows the topography to be reconstructed over a larger lateral field of view and the influence of microsphere imperfections to be reduced (due to the lower sphericity). However, the super-resolving power of the imaging technique is lower [14], thus providing the possibility of measuring a 800 -nm-period grating in air, in this case.

\section{CONCLUSION}

An experimental method is proposed for the combination of microsphere-assisted microscopy with coherentphase-shifting interference microscopy in order to measure $3 \mathrm{D}$ topographies with a high quantitative accuracy. Similar $30-\mu \mathrm{m}$-diameter glass microspheres are introduced in each interference arm to compensate the optical aberration and to further remove the phase distortion from the microbead without the need for image processing. A full-field superresolution system in air, based on a Linnik configuration, makes it possible to perform subwavelength 3D imaging in the far field. Indeed, using a compensating microsphere, a lateral resolution of at least $400 \mathrm{~nm}$ is achieved in air using a 0.55 -numerical-aperture microscope objective and 
quasicoherent illumination. An axial sensitivity of around $4 \mathrm{~nm}$ is reached. High-accuracy quantitative 3D measurements of a reference grating having a line width of 300 $\mathrm{nm}$ and transparent nanopillars are performed. The correct object shapes and the right height distributions are retrieved in air. Without microspheres, the interferometer would require an equivalent numerical aperture of 1.5 , which is relatively complex to implement in interference microscopy. Due to the high numerical aperture of the imaging system, the phase-shift increments must be taken into account. The introduction of a microsphere holder in each interferometer arm in order to position the microspheres and to perform contactless measurements would be a significant research perspective. Moreover, microsphereaberration retrieval would make it possible to reduce the influence of sphere imperfections. Finally, the measurement errors and the repeatability depending on the dissimilarity of the diameter and the refractive index of spheres should be systematically investigated.

\section{ACKNOWLEDGMENTS}

This work received funding from SATT Conectus Alsace and was supported by the University of Strasbourg and by the Deutsche Forschungsgemeinschaft (DFG) through program DFG-SPP 1839, "Tailored Disorder." G.G. acknowledges funding by the Helmholtz Postdoctoral Program and the Karlsruhe School of Optics and Photonics (KSOP). We thank the French RENATECH network (FEMTO-ST institute, France) for fabricating the Ronchi calibration target and Amandine Elchinger for her constructive input in writing the manuscript. We also acknowledge help from the $C^{3}$-Fab platform of the ICube laboratory.

[1] D. Malacara, Optical Shop Testing (John Wiley \& Sons, Hoboken, 2006).

[2] P. Lehmann, W. Xie, B. Allendorf, and S. Tereschenko, Coherence scanning and phase imaging optical interference microscopy at the lateral resolution limit, Opt. Express 26, 7376 (2018).

[3] C. Giusca and R. Leach, Calibration of the scales of areal surface topography measuring instruments: Part 3. Resolution, Meas. Sci. Technol. 24, 105010 (2013).

[4] R. Leach, C. Giusca, A. Henning, B. Sherlock, and J. Coupland, in Fringe 2013, edited by W. Osten (Springer, Berlin, 2014), p. 405.

[5] Geometrical product specifications (GPS) - Surface texture: Areal-Part 600: Metrological characteristics for areal topography measuring methods, ISO 25178-600 (2019).

[6] P. de Groot and X. de Lega, in Fringe 2005, edited by W. Osten (Springer, Berlin, 2006).

[7] J. Goodman, Introduction to Fourier Optics (McGraw Hill, New York, 1996).
[8] R. Horstmeyer, R. Heintzmann, G. Popescu, L. Waller, and C. Yang, Standardizing the resolution claims for coherent microscopy, Nat. Photonics 10, 68 (2016).

[9] P. Lehmann, J. Niehues, and S. Tereschenko, 3-D optical interference microscopy at the lateral resolution limit, Int. J. Optomechatronics 8, 231 (2014).

[10] O. Haeberle, K. Belkebir, H. Giovaninni, and A. Sentenac, Tomographic diffractive microscopy: Basics, techniques and perspectives, J. Mod. Opt. 57, 686 (2010).

[11] Y. Cotte, F. Toy, P. Jourdain, N. Pavillon, D. Boss, P. Magistretti, P. Marquet, and C. Depeursinge, Marker-free phase nanoscopy, Nat. Photonics 7, 113 (2013).

[12] J. Y. Lee, B. H. Hong, W. Y. Kim, S. K. Min, Y. Kim, M. V. Jouravlev, R. Bose, K. S. Kim, I.-C. Hwang, L. J. Kaufman, C. W. Wong, P. Kim, and K. S. Kim, Near-field focusing and magnification through self-assembled nanoscale spherical lenses, Nature 460, 498 (2009).

[13] Z. Wang, W. Guo, L. Li, B. Luk'yanchuk, A. Khan, Z. Liu, $Z$. Chen, and $\mathrm{M}$. Hong, Optical virtual imaging at $50 \mathrm{~nm}$ lateral resolution with a white-light nanoscope, Nat. Commun. 2, 218 (2011).

[14] S. Perrin, S. Lecler, and P. M. Montgomery, in LabelFree Super-Resolution Microscopy, edited by V. Astratov (Springer, Cham, 2019).

[15] S. Perrin, H. Li, S. Lecler, and P. Montgomery, Unconventional magnification behaviour in microsphere-assisted microscopy, Opt. Laser Technol. 114, 40 (2019).

[16] H. Sum Sam Lai, F. Wang, Y. Li, B. Jia, L. Liu, and W. J. $\mathrm{Li}$, Super-resolution real imaging in microsphere-assisted microscopy, PLoS ONE 11, e0165194 (2016).

[17] S. Lecler, S. Perrin, A. Leong-Hoi, and P. Montgomery, Photonic jet lens, Sci. Rep. 9, 4725 (2019).

[18] A. Maslov and V. Astratov, Resolution and Reciprocity in Microspherical Nanoscopy: Point-Spread Function versus Photonic Nanojets, Phys. Rev. Appl. 11, 064004 (2019).

[19] X. Hao, C. Kuang, X. Liu, H. Zhang, and Y. Li, Microsphere based microscope with optical super-resolution capability, Appl. Phys. Lett. 99, 203102 (2011).

[20] Y. Ben-Aryeh, Increase of resolution by use of microspheres related to complex Snell's law, J. Opt. Soc. Am. A 33, 2284 (2016).

[21] A. Darafsheh, G. Walsh, L. D. Negro, and V. Astratov, Optical super-resolution by high-index liquid immersed microspheres, Appl. Phys. Lett. 101, 141128 (2012).

[22] L. Li, W. Guo, Y. Yan, S. Lee, and T. Wang, Label-free super-resolution imaging of adenoviruses by submerged microsphere optical nanoscopy, Light Sci. Appl. 2, e104 (2013).

[23] S. Perrin, H. Li, K. Badu, T. Comparon, G. Quaranta, N. Messaddeq, N. Lemercier, P. Montgomery, J.-L. Vonesch, and S. Lecler, Transmission microsphere-assisted darkfield microscopy, Phys. Status Solidi RRL 13, 1800445 (2019).

[24] F. Wang, L. Liu, P. Yu, Z. Liu, H. Yu, Y. Wang, and W. J. Li, Three-dimensional super-resolution morphology by near-field assisted white-light interferometry, Sci. Rep. 6, 24703 (2016).

[25] S. Perrin, A. Leong-Hoi, S. Lecler, P. Pfeiffer, I. Kassamakov, A. Nolvi, E. Haeggstrom, and P. Montgomery, Microsphere-assisted phase-shifting profilometry, Appl. Opt. 56, 7249 (2017). 
[26] M. Aakhte, V. Abbasian, E. A. Akhlaghi, A.-R. Moradi, A. Anand, and B. Javidi, Microsphere-assisted super-resolved Mirau digital holographic microscopy for cell identification, Appl. Opt. 56, D8 (2017).

[27] R. Leach and B. Sherlock, Applications of super-resolution imaging in the field of surface topography measurement, Surf. Topogr. Metrol. Prop. 2, 023001 (2014).

[28] P. Chavel, Optical noise and temporal coherence, J. Opt. Soc. Am. 70, 935 (1980).

[29] B. Yan, Z. Wang, A. L. Parker, Y.-K. Lai, P. J. Thomas, L. Yue, and J. N. Monks, Superlensing microscope objective lens, Appl. Opt. 56, 3142 (2017).

[30] G. Huszka, H. Yang, and M. A. M. Gijs, Microsphere-based super-resolution scanning optical microscope, Opt. Express 25, 15079 (2017).
[31] J. Li, W. Liu, T. Li, I. Rozen, J. Zhao, B. Bahari, B. Kante, and J. Wang, Microsphere-based super-resolution scanning optical microscope, Nano Lett. 16, 6604 (2016).

[32] G. Schulz and K. Elssner, Errors in phase-measurement interferometry with high numerical apertures, Appl. Opt. 30, 4500 (1991).

[33] H. Takajo and T. Takahashi, Noniterative method of obtaining the exact solution for the normal equation in leastsquares phase estimation from the phase difference, J. Opt. Soc. Am. A 5, 1818 (1988).

[34] Y. J. Donie, M. Smeets, A. Egel, F. Lentz, J. B. Preinfalk, A. Mertens, V. Smirnov, U. Lemmer, K. Bittkau, and G. Gomard, Light trapping in thin film silicon solar cells via phase separated disordered nanopillars, Nanoscale 10, 6651 (2018) 